Mater. Res. Soc. Symp. Proc. Vol. 1479 @ 2012 Materials Research Society

DOI: 10.1557/opl.2012.1589

\title{
Effect of Equivalent Sites on the Dynamics of Bimetallic Nanoparticles
}

\author{
C. Fernández-Navarro ${ }^{1}$, A. J. Gutiérrez-Esparza ${ }^{2}$, J.M. Montejano-Carrizales ${ }^{3}$ and S.J. Mejía- \\ Rosales ${ }^{1}$ \\ ${ }^{1}$ Facultad de Ciencias Físico-Matemáticas, Universidad Autónoma de Nuevo León, San Nicolás \\ de los Garza, Nuevo León, México 66450 \\ ${ }^{2}$ División de Ciencias e Ingenierías, Universidad de Guanajuato, León, Gto. México 37150 \\ ${ }^{3}$ Instituto de Física, Universidad Autónoma de San Luis Potosí, San Luis Potosí, S.L.P., México \\ 78000
}

\begin{abstract}
Using a Sutton and Chen interatomic potential, we study the molecular dynamics of $\mathrm{Au}-$ Pd nanoparticles with an initial icosahedral structure at different temperatures and concentrations, where each relative concentration of the 561-atom particles was made by placing atoms of the same species at equivalent sites, in order to identify under which conditions the melting transition temperature appears for each particle. In addition, we compute global order parameters in order to correlate the obtained results with the caloric curves of each particle. As a result, we observe that the melting transition temperature depends on the relative atomic positions of gold and palladium. The melting transition temperature of the Au-Pd alloy particles appears at higher temperature than that of the pure-gold particle. From the analysis of the structure of the particles, we found that the melting temperature increases with the proportion of gold atoms, and for those particles with a higher concentration of palladium on the surface, we observe an early migration of gold atoms before the melting transition temperature appears.
\end{abstract}

Keywords: Nanoparticles, gold, palladium, molecular dynamics.

\section{INTRODUCTION}

The uses of nanoparticles have grown in a large measure both in number and scope over the last decade. Besides their use as catalysts, metallic nanoparticles are very promising agents in the development of electronic devices. Nowadays the application of nanostructured materials as protein detectors [1] contrast enhancers in imaging techniques [1], catalysts [2], drug delivery [3], and antibacterial agents [4], has become of great importance, mainly due to their stability and selectivity features. As applications of nanoparticles grow in different and sometimes unexpected directions, the need of a better understanding of the influence of structure on the properties of the particles becomes more accentuated, specially when the choice of a method of synthesis is determined by how the final structure and size of the nanoparticle depend on the chosen technique [5-7]. Synthesis is possible with chemical based methods to obtain colloidal dispersions of nanoparticles [8] and physical methods of deposition, where the nanoparticles are formed by high-pressure sputtering in a controlled gas flow atmosphere, can be used to generate particles with narrow size distributions and, more important, with well defined chemical composition $[9,10]$.

Along with the variety in the methods of synthesis, characterization techniques have been improving constantly. One of the most important tools in the analysis of nanostructures is highresolution transmission electron microscopy (HRTEM) [11]; with HRTEM is possible to study 
simultaneously the structure and the chemical composition of the particles with a high degree of resolution. By the use of numerical methods is possible to make the reconstruction of electron exit waves from focal series of lattice images, what extends the HRTEM resolution to fractions of Angstrom, allowing imaging the atomic structure of the nanoparticle directly. This resolution capability potentiates the in-depth study of the geometrical characteristics of the nanoparticles, and their differences with respect to the crystal structure of the bulk.

Experimental and theoretical studies have been made regarding the structural properties and formation of metal nanoparticles. The structure of a nanoparticle depends on the method of synthesis used as well as on the thermal treatment to which the particle turns out to be subject. Hence, we can infer that it is important to count with studies that allow the understanding of the structural and energy changes that happen in the particle as its temperature is varied. Similar works have studied the melting and freezing of nanoparticles at different geometries [12-14]. The purpose of this work is to contribute in a certain extent to improve the understanding of the thermodynamic characteristics of metallic and bimetallic nanoparticles, emphasizing on the surface phenomenology of the particles, since the practical uses of these systems will depend on the behavior and physical properties of the surface.

In this work, we have chosen nanoparticles with icosahedral geometry as starting configurations, since there is wide experimental evidence that the icosahedral geometry is one of the most energetically geometries favorable for metallic particles at this range of sizes [8]. An icosahedron is a geometric structure with 12 vertices connected by 30 edges forming 20 triangular faces. According to their positions in the particle, different atoms occupy identical geometric sites, called equivalent sites. The atoms located in equivalent sites have the same type and number of neighbors, and are placed at the same distance from the origin [15]. We chose an icosahedral nanoparticle of 561 atoms at 5 different concentrations of gold and palladium, where every concentration remained determined by the election of the equivalent sites where the atoms were placed depending on their atomic species, as can be seen in figure 1 . We studied the conditions of the melting and freezing transitions for each of these particles.
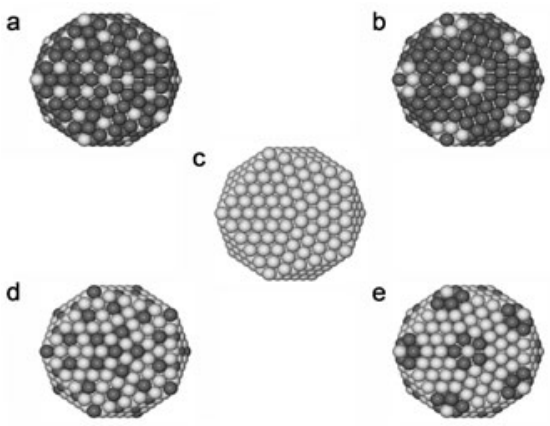

Figure 1. Models that correspond to the initial configurations of the particles with concentrations of (a) $\mathrm{Au}_{261} \mathrm{Pd}_{300}$, (b) $\mathrm{Au}_{271} \mathrm{Pd}_{290}$, (c) $\mathrm{Au}_{561}$, (d) $\mathrm{Pd}_{261} \mathrm{Au}_{300}$ and (e) $\mathrm{Pd}_{271} \mathrm{Au}_{290}$. The white spheres represent gold atoms, whereas the dark spheres represent palladium atoms. 


\section{SIMULATION DETAILS}

Using a Sutton-Chen potential model [16] to model the interactions in the Au-Pd bimetallic nanoparticles, we performed the Molecular Dynamics simulations in the canonical ensemble (NVT) making use of the Nosé-Hoover thermostat [17, 18], and following the same strategy used in a previous set of simulations of Au-Pd nanoparticles [19]. Sutton and Chen modified the Finnis-Sinclair potential to study the interactions between particles, and developed it for ten fcc metal species:

$$
U(r)=\varepsilon\left[\frac{1}{2} \sum_{j \neq i}\left(\frac{a}{r_{i j}}\right)^{n}-c \sqrt{\rho_{i}}\right],
$$

where

$$
\rho_{i}=\sum_{j \neq i}\left(\frac{a}{r_{i j}}\right)^{m} .
$$

In the equations 1 and 2, the parameters $m$ and $n$ are positive integers, $\varepsilon$ is a parameter with energy dimensions, $a$ is a fcc lattice constant, and $c$ is an adimensional positive parameter that fits according to experimental results.

For every simulation, we used 500,000 time steps, of which 100,000 were used to reach thermal equilibrium and were not considered in the analysis of the results; using a time step of $0.0015 \mathrm{ps}$ we performed a set of NVT simulations, each $750 \mathrm{ps}$ long, for every nanoparticle. For every particle, we performed two series of simulations. In the first series, corresponding to the heating process, we covered a range of temperature from 300 to $1200 \mathrm{~K}$, increasing $T$ every 20 $\mathrm{K}$, using as the initial configuration for every temperature, the final configuration obtained at a previous temperature. In the second series, or cooling process, the swept on temperature was made backing down in temperature from 1200 to $300 \mathrm{~K}$.

To identify the nanoparticles we use the notation $\mathrm{Au}_{\mathrm{X}} \mathrm{Pd}_{\mathrm{Y}}$ to refer to a particle of $X$ gold atoms and $Y$ palladium atoms.

\section{RESULTS}

Structural phase transitions were recognized for every particle at both heating and cooling processes, by analyzing the evolution of the energy during the simulation, and by the analysis of the structure of the particles. The melting temperature for $\mathrm{Au}_{561}$ was $660 \mathrm{~K} \pm 10 \mathrm{~K}, 940 \mathrm{~K} \pm 10 \mathrm{~K}$ for $\mathrm{Au}_{271} \mathrm{Pd}_{290}, 900 \mathrm{~K} \pm 10 \mathrm{~K}$ for $\mathrm{Au}_{261} \mathrm{Pd}_{300}, 940 \mathrm{~K} \pm 10 \mathrm{~K}$ for $\mathrm{Pd}_{271} \mathrm{Au}_{290}$ and $960 \mathrm{~K} \pm 10 \mathrm{~K}$ for $\mathrm{Pd}_{261} \mathrm{Au}_{300}$. The melting and freezing temperatures can be identified by the abrupt changes in the slope of the caloric curves, as can be seen in figure 2. We found that for the $\mathrm{Au}_{261} \mathrm{Pd}_{300}$ and $\mathrm{Au}_{271} \mathrm{Pd}_{290}$ particles, at temperatures between $700 \mathrm{~K}$ and $800 \mathrm{~K}$ the energy decreases with the temperature, while for $\mathrm{Pd}_{271} \mathrm{Au}_{290}$ and $\mathrm{Pd}_{261} \mathrm{Au}_{300}$ at temperatures greater than $820 \mathrm{~K} \pm 10 \mathrm{~K}$ the energy for this particles increases, due to structural changes before at the melting point, mainly at the surface of the particles. Figure 3 shows snapshots of the particles at different temperatures during the heating process. 

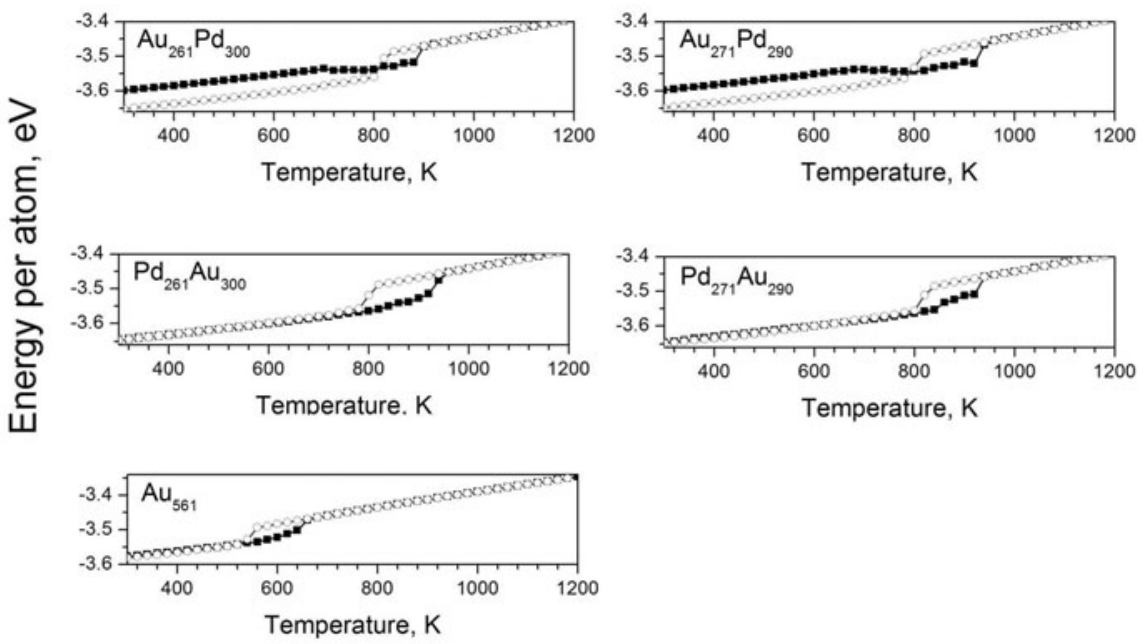

Figure 2. Configuration energy vs. temperature for the particles (a) $\mathrm{Au}_{261} \mathrm{Pd}_{300}$, (b) $\mathrm{Au}_{271} \mathrm{Pd}_{290}$, (c) $\mathrm{Pd}_{261} \mathrm{Au}_{300}$, (d) $\mathrm{Pd}_{271} \mathrm{Au}_{290}$ and (e) $\mathrm{Au}_{561}$. The dark and light symbols correspond to the heating and cooling processes, respectively.

By the knowledge of the original sites of both atomic species, and analyzing the radial distribution function (RDF), calculated using the center of mass as the origin, we found that in the $\mathrm{Au}_{261} \mathrm{Pd}_{300}$ and its counterpart $\mathrm{Pd}_{261} \mathrm{Au}_{300}$, the reversal in the structural role of the atomic species has a very appreciable effect: the $\mathrm{Au}_{261} \mathrm{Pd}_{300}$ particle has nucleus rich in gold, and its radius is $7 \AA$, while the $\mathrm{Pd}_{261} \mathrm{Au}_{300}$ has a nucleus mainly made of $\mathrm{Pd}$, and its radius is $6 \AA$; this remarked difference in the composition of the cores is the main cause that these particles have very pronounced difference in the values of their melting temperatures, since a larger amount of thermal energy is needed for a Pd immersed in the core to migrate out of its original site. This is also the reason why Au atoms have a marked tendency to migrate to the surface of the particle once the thermal energy is enough to allow diffusion. For purposes of comparison, we show in figure 4 the $\mathrm{RDF}$ at $300 \mathrm{~K}$ (quite well before melting), and at a temperature just before melting (between 900 and 960K), when diffusion has already started. Right after melting, for all the particles the composition of gold at the surface becomes larger than at the beginning of the heating process, and larger than the concentration of palladium. 
a)
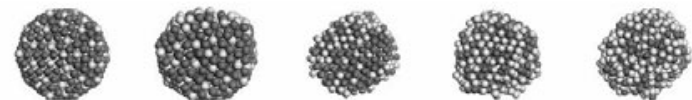

$300 \mathrm{~K}$

$700 \mathrm{~K}$

$800 \mathrm{~K}$

$880 \mathrm{~K}$

$900 \mathrm{~K}$

b)
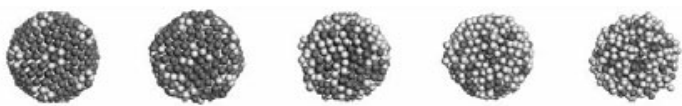

$300 \mathrm{~K}$

$700 \mathrm{~K}$

$840 \mathrm{~K}$

$920 \mathrm{~K}$

$940 \mathrm{~K}$

c)
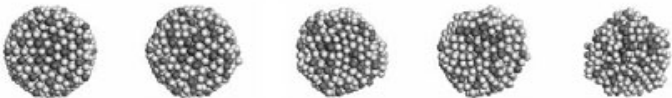

$300 \mathrm{~K}$

$700 \mathrm{~K}$

$880 \mathrm{~K}$

$920 \mathrm{~K}$

$940 \mathrm{~K}$

d)
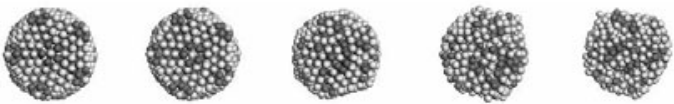

$300 \mathrm{~K}$

$720 \mathrm{~K}$

$840 \mathrm{~K}$

$920 \mathrm{~K}$

$940 \mathrm{~K}$

e)
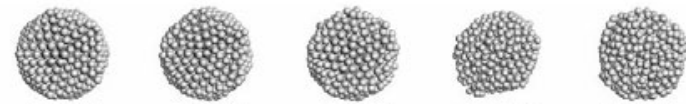

$300 \mathrm{~K}$

$520 \mathrm{~K}$

$580 \mathrm{~K}$

$640 \mathrm{~K}$

$660 \mathrm{~K}$

Figure 3. Structural modification of the nanoparticles in the heating process. (a) $\mathrm{Au}_{261} \mathrm{Pd}_{300}$; (b) $\mathrm{Au}_{271} \mathrm{Pd}_{290}$; (c) $\mathrm{Pd}_{261} \mathrm{Au}_{300}$; (d) $\mathrm{Pd}_{271} \mathrm{Au}_{290}$; and (e) $\mathrm{Au}_{561}$.

To investigate the structural behavior of the particles during the heating and cooling processes, we calculated the global order parameter $\mathrm{Q}_{6}$ which is defined as [20]

$$
Q_{6}(i)=\left(\frac{4 \pi}{2 l+1} \sum_{m=-l}^{l}\left|\bar{Q}_{6 m}(i)\right|^{2}\right)^{\frac{1}{2}} \text {, }
$$

where

$$
\bar{Q}_{6 m}(i)=\frac{\sum_{i=1}^{N} N_{n b}(i) q_{6 m}(i)}{\sum_{i=1}^{N} N_{n b}(i)} .
$$



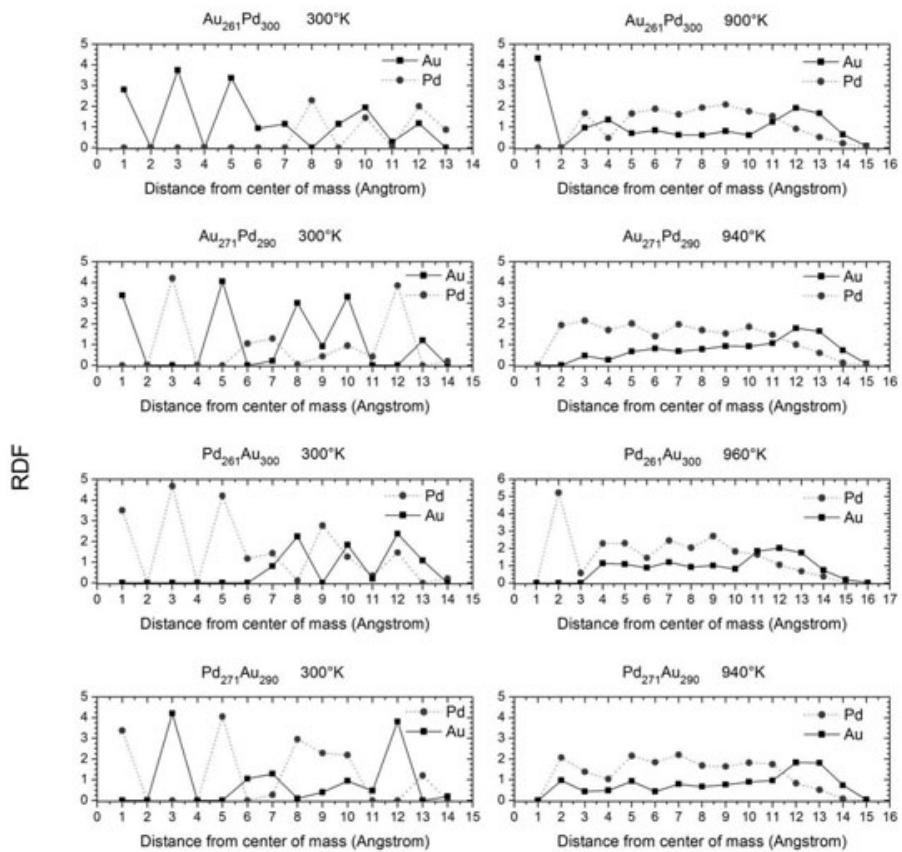
melting.

Figure 4. $\mathrm{Pd}$ and Au radial distributions, at $300 \mathrm{~K}$, and at a temperature just before

$N$ is the total number of atoms in the particle, $N_{n b}(i)$ is the number of first neighbors for the $i$-th atom, and $q_{6 m}(i)$ measures the local order around the $i$-th atom considering the average of the spherical harmonics $Y_{6 m}$ of the bonds with the $N_{n b}(i)$ neighbors:

$$
q_{6 m}(i)=\frac{\sum_{j=1}^{N_{n b}} Y_{6 m}\left(r_{i j}\right)}{N_{n b}(i)} .
$$

Defined in this way, the order parameter $\mathrm{Q}_{6}$ becomes a fair criteria to identify the resulting structures of the heating process, because a specific structure is characterized by welldefined value of $\mathrm{Q}_{6}$ : a perfect $\mathrm{FCC}$ structure gives a value $\mathrm{Q}_{6}{ }^{\mathrm{FCC}}=0.575$, whereas an icosahedron gives $\mathrm{Q}_{6}{ }^{\mathrm{Th}}=0.663$, while for melted states, the value of $\mathrm{Q}_{6}$ will drop down to values close to zero. The cutoff distance for identifying the nearest neighbors was taken to be $3.6 \mathrm{~A}$ at $300 \mathrm{~K}$. This corresponds to the position of the first minimum in the pair correlation function for fcc Pd-Au. Figure 5 shows the variation in the order parameter with temperature for the nanoparticles. 

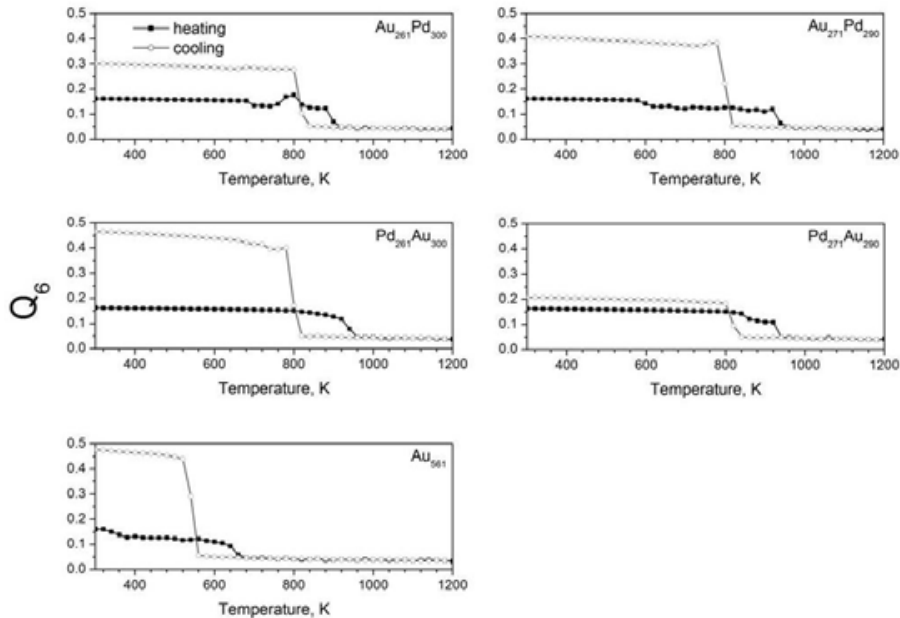

Figure 5. Global order parameter $\mathrm{Q}_{6}$ vs the temperature for the five different concentrations used in this study.

The final geometries of the particles after the heating-cooling process in general differ from the original configurations, and from the calculation of $\mathrm{Q}_{6}$ we found that these difference are not just in the shape, but on the overall atomic ordering in the volume of the particle. Only the $\mathrm{Pd}_{271} \mathrm{Au}_{290}$ and $\mathrm{Au}_{261} \mathrm{Pd}_{300}$ particles freeze taking an icosahedral shape, while the other particles crystallize as truncated octahedra. The $\mathrm{Pd}_{271} \mathrm{Au}_{290}$ particle show a larger stability than its $\mathrm{Au}_{271} \mathrm{Pd}_{290}$ counterpart, that experiences structural changes at temperatures as low as $680 \mathrm{~K}$, even when both particles have a very similar melting temperature. Again, this is mostly due to the difference in the amount of Pd between the particles. A similar trend can be noted in a slightly diminished way between the $\mathrm{Au}_{261} \mathrm{Pd}_{300}$ and the $\mathrm{Pd}_{261} \mathrm{Au}_{300}$ particles.

\section{CONCLUSION}

Gold atoms diffuse towards the surface before the melting temperature for all particles, in agreement with other works $[19,21]$. As a result, high concentrations of palladium are not observed in the surface. In the heating curve of the $\mathrm{Au}_{561}$ nanoparticle, we see a gradual change in the energy before the melting transition happens. This happens because the atoms in the surface melt before the other ones composing the nanoparticle. For the $\mathrm{Pd}_{261} \mathrm{Au}_{300}$ and $\mathrm{Pd}_{271} \mathrm{Au}_{290}$ particles, migration of $\mathrm{Au}$ is not necessary in order to minimize the surface energy if gold is present on the surface before thermal equilibrium is. As soon as the heating - cooling cycle is closed, the particle $\mathrm{Au}_{290} \mathrm{Pd}_{271}$ returns to its original values of potential energy at room temperature, which can be understood as an evidence that the icosahedral geometry is energetically suited for this particle. We observed that the melting transition temperature 
depends on the relative atomic positions of gold and palladium. We found that the melting transition temperature of the Au-Pd alloy particles appears at higher temperature than that of the pure-gold particle. From the analysis of the structural succession of the particles, we found that the melting temperature increases because of the presence of gold, and for those particles with a higher concentration of palladium on the surface, we observe an early migration of gold atoms before the melting transition temperature appears.

\section{ACKNOWLEDGMENTS}

Support from the Mexican Council for Science and Technology (CONACYT, Mexico), through project CIAM 148967, is acknowledged.

\section{REFERENCES}

1. O. V. Salata, Nanobiotechnology 2 (3), 1-6 (2004).

2. M. O. Nutt, J. B. Hughes and M. S. Wong, Environ. Sci. Technol. 39 (5) 1346 (2005).

3. I. Bala, S. Hariharan and M.N. Kumar, Crit. Rev. Ther. Drug Carrier Syst. 21 (5), 387 (2004).

4. J.R. Morones, J. L. Elechiguerra, A. Camacho, K. Holt, J. B. Kouri, J. Tapia-Ramírez, and M. José-Yacamán, Nanotechnology 16, 2346 (2005).

5. M.-L. Wu, D.-H. Chen and T.-C. Huang, Langmuir 17 (13), 3877 (2001).

6. F.-R. Fan, D.-Y. Liu, Y.-F. Wu, S. Duan, Z.-X. Xie, Z.-Y. Jiang, and Z.-Q. Tian. J. Am. Chem. Soc. 130, 6949 (2008).

7. D.-H. Chen and C.-J. Chen. J. Mater. Chem. 12, 1557 (2002).

8. R. Ferrando, J. Jellinek and R.L. Johnston, Chem. Rev. 108, 845 (2008).

9. S. Tajammul Hussain, M. Iqbal and M. Mazhar, J. Nanopart. Res. 11, 1383 (2008).

10. H. Qian and R. Jin, Nano Lett. 9, 4083 (2009).

11. M. M. Mariscal, O.A. Oviedo, E.P.M. Leiva, S. Mejía-Rosales and M. José-Yacamán, Nanostructure Science and Technology 3 (26) (2013).

12. J. Shim, B. Lee and Y.W. Cho, Surface Science 512, 262 (2002).

13. Y. Shibuta and T. Suzuki, Chemical Physics Letters 445, 265 (2007).

14. Y. Shibuta and T. Suzuki, Chemical Physics Letters 498, 323 (2010).

15. J.M. Montejano-Carrizales and J.L. Morán-López, Nanostructured Materials 1, 397 (1992).

16. H. Rafii-Tabar and A.P. Sutton, Philosophical Magazine Letters 63, 217 (1991).

17. S. Nosé, Molecular Physics 52, 255 (1984).

18. W.G. Hoover, Phys. Rev. A 31, 1695 (1985).

19. S. J. Mejía-Rosales, C. Fernández-Navarro, E. Pérez-Tijerina, J. M. Montejano-Carrizales and M. José-Yacamán, J. Phys. Chem. B 110, 12884 (2006).

20. Y. Chushak and L.S. Bartell, European Physical Journal D 16, 43 (2001).

21. F Pittaway, L. O. Paz-Borbón, R. L. Johnston, H. Arslan, R. Ferrando, C. Mottet, G. Barcaro, and A. Fortunelli, J. Phys. Chem. C 113, 9141 (2009). 